\title{
Banach spaces with a supershrinking basis
}

by

\author{
GINÉS LÓPEZ (Granada)
}

Abstract. We prove that a Banach space $X$ with a supershrinking basis (a special type of shrinking basis) without $c_{0}$ copies is somewhat reflexive (every infinite-dimensional subspace contains an infinite-dimensional reflexive subspace). Furthermore, applying the $c_{0}$-theorem by Rosenthal, it is proved that $X$ contains order-one quasireflexive subspaces if $X$ is not reflexive. Also, we obtain a characterization of the usual basis in $c_{0}$.

1. Introduction. We use standard Banach space facts and terminology. Let us just recall that a basis $\left\{e_{n}\right\}$ in a Banach space $X$ is called shrinking iff $X^{*}=\varlimsup \ln \left\{f_{n}: n \in \mathbb{N}\right\}$, where $\left\{f_{n}\right\}$ is the sequence of biorthogonal functionals.

With a separation argument, it is easy to see that $\left\{e_{n}\right\}$ is shrinking iff $\left\{x^{* *} \in X^{* *}: x^{* *}\left(f_{n}\right)=0 \forall n \in \mathbb{N}\right\} \subset X$.

We will say that $\left\{e_{n}\right\}$ is supershrinking iff

$$
\left(N_{X}=\right)\left\{x^{* *} \in X^{* *}: \lim _{n} x^{* *}\left(f_{n}\right)=0\right\} \subset X .
$$

In fact, if the basis $\left\{e_{n}\right\}$ is seminormalized, one can replace the above inclusion by equality. Also, it is possible give a definition only in terms of the basis: a shrinking basis $\left\{e_{n}\right\}$ is supershrinking provided whenever scalars $\left\{c_{n}\right\}$ satisfy $\lim _{n} c_{n}=0$ and $\sup _{n}\left\|\sum_{k=1}^{n} c_{k} e_{k}\right\|$ is finite, then $\sum_{n} c_{n} e_{n}$ converges in the norm topology.

In [4], it was proved that the Radon-Nikodym and Krein-Milman properties are equivalent in Banach spaces with a supershrinking basis. Also, the supershrinking bases are used in [6] to prove that every Banach space with the point of continuity property and separable dual is somewhat order-one quasireflexive (every non-reflexive subspace contains an order-one quasireflexive subspace). A Banach space is order-one quasireflexive if it has codimension one in its bidual.

1991 Mathematics Subject Classification: Primary 46B03.

Supported by D.G.E.S. Project No. 96-1406. 
Examples of Banach spaces with a supershrinking basis are the reflexive spaces with basis, $c_{0}, J$ (the James space) and the natural predual of $J T$ (the James tree space) (see [4]). In a similar way, it can be seen that the natural predual of $J T_{\infty}$ has a supershrinking basis (see Th. IV.2 in [2]).

The aim of this note is to study the structure of Banach spaces with a supershrinking basis. Our main results are the following:

THEOREM A. Let $X$ be a Banach space with a normalized and shrinking basis $\left\{e_{n}\right\}$, and associated functionals $\left\{f_{n}\right\}$, without infinite-dimensional reflexive subspaces. Then the following are equivalent:

(i) $\left\{e_{n}\right\}$ is supershrinking.

(ii) $N_{X}$ is separable.

(iii) $A$ w-closed and bounded subset $K$ of $X$ is w-compact whenever $\lim _{n} f_{n}(k)=0$ uniformly in $k \in K$.

(iv) $A$ closed and bounded subset $K$ of $X$ is compact whenever $\lim _{n} f_{n}(k)$ $=0$ uniformly in $k \in K$.

(v) $\left\{e_{n}\right\}$ is equivalent to the usual basis of $c_{0}$.

THEOREM B. Let $X$ be a non-reflexive Banach space with a normalized supershrinking basis $\left\{e_{n}\right\}$ and associated functionals $\left\{f_{n}\right\}$, without subspaces isomorphic to $c_{0}$. Then $X$ contains order-one quasireflexive subspaces. In fact, every non-reflexive subspace contains an order-one quasireflexive subspace.

Main results. We begin by recalling some concepts and known results. A basic sequence $\left\{x_{n}\right\}$ in a Banach space $X$ is said to be strongly sum$\operatorname{ming}$ (s.s.) if $\left\{x_{n}\right\}$ is weakly Cauchy and

$$
\sum_{n} c_{n} \text { converges whenever } \sup _{n}\left\|\sum_{k=1}^{n} c_{k} x_{k}\right\|<\infty .
$$

The usual basis of $c_{0}$ is the basis of unit vectors denoted by $\left\{b_{n}\right\}$, and the summing basis of $c_{0}$ is $\left\{\sum_{i=1}^{n} b_{i}\right\}_{n}$.

THEOREM ([5], $c_{0}$-Rosenthal). Let $X$ be a Banach space and $\left\{x_{n}\right\}$ a weakly Cauchy and not weakly convergent sequence in $X$. Then $\left\{x_{n}\right\}$ has either a strongly summing basic subsequence or a convex basic block equivalent to the summing basis of $c_{0}$.

THEOREM ([1], Elton). Let $\left\{x_{n}\right\}$ be a normalized and weakly null sequence in a Banach space, without subsequences equivalent to the usual basis of $c_{0}$. Then $\left\{x_{n}\right\}$ has a basic subsequence $\left\{x_{n}^{\prime}\right\}$ such that $\lim _{n}\left\|\sum_{k=1}^{n} c_{i} y_{i}\right\|$ $=\infty$ for every subsequence $\left\{y_{n}\right\}$ of $\left\{x_{n}^{\prime}\right\}$ and for every $\left\{c_{n}\right\} \notin c_{0}$.

Our first result characterizes the equality $N_{X}=X$.
THEOREM 1. Let $X$ be a Banach space with a normalized and shrinking basis $\left\{e_{n}\right\}$ and associated functionals $\left\{f_{n}\right\}$. Then the following are equivalent:

(i) $N_{X} \neq X$.

(ii) There is a non-reflexive subspace $Y$ of $X$ with $\lim _{n}\left\|f_{n \mid Y}\right\|_{Y^{*}}=0$.

(iii) There is a bounded and non-relatively weakly compact subset $K$ of $X$ such that $\lim _{n} \sup _{k \in K} f_{n}(k)=0$.

Proof. (i) $\Rightarrow$ (ii). Let $x_{0}^{* *} \in N_{X} \backslash X$. Then the sequence $\left\{z_{n}\right\}=$ $\left\{\sum_{k=1}^{n} x_{0}^{* *}\left(f_{k}\right) e_{k}\right\}_{n}$ is weakly Cauchy and not weakly convergent. In fact, it converges to $x_{0}^{* *}$ in $X^{* *}$, for the weak-* topology.

By applying the $c_{0}$-Rosenthal Theorem, either there is an increasing $\sigma: \mathbb{N} \rightarrow \mathbb{N}$ such that $\left\{z_{\sigma(n)}\right\}$ is a basic strongly summing sequence, or $\left\{z_{n}\right\}$ has a convex basic block equivalent to the summing basis. In the first case we set $y_{n}=\sum_{k=1}^{\sigma(n)} x_{0}^{* *}\left(f_{k}\right) e_{k}$ for $n \in \mathbb{N}$.

Assume, without loss of generality, that $x_{0}^{* *}\left(f_{n}\right) \neq 0$ for every $n \in \mathbb{N}$.

We put $Y=\varlimsup\left\{y_{n}: n \in \mathbb{N}\right\}$ and let $\left\{g_{n}\right\}$ be the sequence of associated functionals of $\left\{y_{n}\right\}$. Then

$$
g_{n}=\frac{f_{\sigma(n) \mid Y}}{x_{0}^{* *}\left(f_{\sigma(n)}\right)}-\frac{f_{\sigma(n+1) \mid Y}}{x_{0}^{* *}\left(f_{\sigma(n+1)}\right)} \quad \forall n \in \mathbb{N} .
$$

Furthermore, $x_{0}^{* *} \in Y^{* *}$. So, $Y$ is not reflexive.

If $y^{* *} \in Y^{* *}$, then $\sup _{n}\left\|\sum_{k=1}^{n} y^{* *}\left(g_{k}\right) y_{k}\right\|<\infty$ and so the series $\sum_{n} y^{* *}\left(g_{n}\right)$ converges since $\left\{y_{n}\right\}$ is strongly summing. But

$$
\sum_{n=1}^{N} y^{* *}\left(g_{n}\right)=\frac{y^{* *}\left(f_{\sigma(1) \mid Y}\right)}{x_{0}^{* *}\left(f_{\sigma(1)}\right)}-\frac{y^{* *}\left(f_{\sigma(N+1) \mid Y}\right)}{x_{0}^{* *}\left(f_{\sigma(N+1)}\right)} \quad \forall N \in \mathbb{N}
$$

Hence, the following limit exists for every $y^{* *} \in Y^{* *}$ :

$$
\lim _{n \rightarrow \infty} \frac{y^{* *}\left(f_{\sigma(n) \mid Y}\right)}{x_{0}^{* *}\left(f_{\sigma(n)}\right)} .
$$

Let $v_{n}=\sum_{k=\sigma(n-1)+1}^{\sigma(n)} x_{0}^{* *}\left(f_{k}\right) e_{k}$ for $n \in \mathbb{N}(\sigma(0)=0)$. Then $Y=\overline{\operatorname{lin}}\left\{v_{n}\right.$ : $n \in \mathbb{N}\}$ and $\left\{v_{n}\right\}$ is a basic block of $\left\{e_{n}\right\}$, so it is a shrinking basis of $Y$. Let $\left\{h_{n}\right\}$ be the sequence of associated functionals of $\left\{v_{n}\right\}$. Then $h_{n}=$ $f_{k \mid Y} / x_{0}^{* *}\left(f_{k}\right)$ whenever $\sigma(n-1)+1 \leq k \leq \sigma(n)$ and $x_{0}^{* *}\left(f_{k}\right) \neq 0$. But $\sup _{n}\left\|h_{n}\right\|<\infty$ by (1). So, $\lim _{n}\left\|f_{n \mid Y}\right\|_{Y^{*}}=0$, and $Y$ is non-reflexive since $x_{0}^{* *} \in Y^{* *}$.

Now, assume that $\left\{z_{n}\right\}$ has a convex basic block equivalent to the summing basis. Then there is an increasing $\sigma: \mathbb{N} \rightarrow \mathbb{N}$ and a sequence $\left\{\lambda_{n}\right\}$ of positive real numbers with $\sum_{k=\sigma(n-1)+1}^{\sigma(n)} \lambda_{k}=1$ for every $n \in \mathbb{N}$ such that $v_{n}=\sum_{k=\sigma(n-1)+1}^{\sigma(n)} \lambda_{k} z_{k}$ is equivalent to the summing basis. 
Let $Y=\varlimsup \operatorname{lin}\left\{v_{n}: n \in \mathbb{N}\right\}$ and let $\left\{g_{n}\right\}$ be the sequence of associated functionals of $\left\{v_{n}\right\}$. Then

(2) $\quad \lambda_{k} g_{n}=\frac{f_{k \mid Y}}{x_{0}^{* *}\left(f_{k}\right)}-\frac{f_{k+1 \mid Y}}{x_{0}^{* *}\left(f_{k+1}\right)} \quad$ whenever $\sigma(n-1)+1 \leq k \leq \sigma(n)$.

Therefore $M_{1}=\sup _{n}\left\|g_{n}\right\|<\infty$.

Let $y_{1}=v_{1}$ and $y_{n}=v_{n}-v_{n-1}$ for $n \geq 2$. Then $\left\{y_{n}\right\}$ is a basis of $Y$ equivalent to the usual basis of $c_{0}$, and

$$
y_{n}=\sum_{k=\sigma(n-1)+1}^{\sigma(n)} \lambda_{k} z_{k}-\sum_{k=\sigma(n-2)+1}^{\sigma(n-1)} \lambda_{k} z_{k} \quad \forall n \geq 2 .
$$

Let $\left\{h_{n}\right\}$ be the sequence of associated functionals of $\left\{y_{n}\right\}$. Then $h_{n}=$ $f_{\sigma(n-1)+1 \mid Y} / x_{0}^{* *}\left(f_{\sigma(n-1)+1}\right)$ for every $n \in \mathbb{N}$ and $M_{2}=\sup _{n}\left\|h_{n}\right\|$ is finite.

By (2), we obtain

$\frac{f_{k+1 \mid Y}}{x_{0}^{* *}\left(f_{k+1}\right)}=\frac{f_{k \mid Y}}{x_{0}^{* *}\left(f_{k}\right)}-\lambda_{k} g_{n} \quad$ whenever $\sigma(n-1)+1 \leq k \leq \sigma(n)$.

Inductively, we have

$$
\frac{f_{k \mid Y}}{x_{0}^{* *}\left(f_{k}\right)}=h_{n}-g_{n} \sum_{i=\sigma(n-1)+1}^{k-1} \lambda_{i} \quad \text { whenever } \sigma(n-1)+2 \leq k \leq \sigma(n)+1 \text {. }
$$

So, $\left\|f_{k \mid Y} / x_{0}^{* *}\left(f_{k}\right)\right\|_{Y^{*}} \leq M_{2}+M_{1}$ for every $k \in \mathbb{N}$ and hence $\lim _{n \rightarrow \infty}\left\|f_{n \mid Y}\right\|_{Y^{*}}$ $=0$.

(ii) $\Rightarrow$ (iii). This is clear, by considering $K=B_{Y}$.

(iii) $\Rightarrow$ (i). Let $k^{* *} \in \bar{K}^{w^{*}} \backslash K$. Then $k^{* *} \in X^{* *} \backslash X$ and $\lim _{n} k^{* *}\left(f_{n}\right)=0$, since $\lim _{n} \sup _{k \in K} f_{n}(k)=0$. So, $y^{* *} \in N_{X} \backslash X$ and $N_{X} \neq X$.

To prove Theorem A we need the following lemmas:

LEMMA 2. Let $X$ a Banach space with a normalized basis $\left\{e_{n}\right\}$ and let $y_{p}=\sum_{n=1}^{\infty} a_{n}^{p} e_{n}$ for every $p \in \mathbb{N}$ be a sequence in $X$ such that $\lim _{k} a_{n}^{k}=0$ uniformly in $n \in \mathbb{N}$ and $\left\|y_{p}\right\| \geq 1$ for every $p \in \mathbb{N}$. Then there is a basic subsequence $\left\{y_{\tau(p)}\right\}$ of $\left\{y_{p}\right\}$ equivalent to a basic block $v_{n}=\sum_{k=\sigma(n-1)+1}^{\sigma(n)} \lambda_{k} e_{k}$ for every $n \in \mathbb{N}$ such that $\inf _{n}\left\|v_{n}\right\|>0$ and $\lim _{k} \lambda_{k}=0$.

Proof (analogous to Prop. 1.a.11 of [3]). Let us see that for every $\varepsilon>0$ and $p \in \mathbb{N}$ there is $k_{p} \in \mathbb{N}$ such that $\left\|\sum_{n=1}^{p} a_{n}^{k_{p}} e_{n}\right\|<\varepsilon$.

If $\varepsilon>0$ and $p \in \mathbb{N}$, then there is $k_{0} \in \mathbb{N}$ such that $\left|a_{n}^{k}\right|<\varepsilon / p$ whenever $k \geq k_{0}$ and $1 \leq n \leq p$. Then

$$
\left\|\sum_{n=1}^{p} a_{n}^{k} e_{n}\right\| \leq \sum_{n=1}^{p}\left|a_{n}^{k}\right|<\varepsilon \quad \forall k \geq k_{0}
$$

Hence it suffices to put $k_{p}=k \geq k_{0}$.
Now, we construct $\left\{y_{\tau(p)}\right\}$ and $\left\{v_{n}\right\}$ inductively. Put $\tau(1)=1$ and $y_{1}=$ $\sum_{n=1}^{\infty} a_{n}^{1} e_{n}$.

Let $p_{1} \in \mathbb{N}$ be such that $\left\|y_{1}-v_{1}\right\|<\frac{1}{4} M$, where $v_{1}=\sum_{n=1}^{p_{1}} a_{n}^{1} e_{n}$ and $M$ is the basic constant of $\left\{e_{n}\right\}$.

Let $\tau(2) \in \mathbb{N}$ be such that $\tau(2)>\tau(1)$ and $\left\|\sum_{n=1}^{p_{1}} a_{n}^{\tau(2)} e_{n}\right\|<\frac{1}{2} \frac{1}{4^{2}} M$ and let $p_{2} \in \mathbb{N}$ with $p_{2}>p_{1}$ and $\left\|\sum_{n=p_{2}+1}^{\infty} a_{n}^{r(2)} e_{n}\right\|<\frac{1}{2} \frac{1}{4^{2}} M$.

Then $\left\|y_{\tau(2)}-v_{2}\right\|<\frac{1}{4^{2}} M$, where $v_{2}=\sum_{n=p_{1}+1}^{p_{2}} a_{n}^{\tau(2)} e_{n}$.

In this way we obtain a basic block $\left\{v_{n}\right\}$ equivalent to $\left\{y_{\tau(n)}\right\}$. So, $\inf _{n}\left\|v_{n}\right\|>0$.

Furthermore, if $v_{n}=\sum_{k=\sigma(n-1)+1}^{\sigma(n)} \lambda_{k} e_{k}$ for every $n \in \mathbb{N}$, then $\lambda_{k}=a_{k}^{\tau(n)}$ whenever $\sigma(n-1)+1 \leq k \leq \sigma(n)$.

So, $\lim _{k} \lambda_{k}=0$, since $\lim _{k} a_{n}^{k}=0$ uniformly in $n \in \mathbb{N}$.

LEMMA 3. Let $X$ be a Banach space with a seminormalized supershrinking basis $\left\{e_{n}\right\}$. If $X$ does not contain infinite-dimensional reflexive subspaces, then $\left\{e_{n}\right\}$ is equivalent to the usual basis of $c_{0}$.

Proof. Let $\left\{f_{n}\right\}$ be the sequence of biorthogonal functionals. Assume that $\left\{e_{n}\right\}$ is not equivalent to the usual basis $\left\{b_{n}\right\}$ of $c_{0}$. We put

$$
X_{0}=\left\{\left\{a_{n}\right\} \in \mathbb{R}^{\mathbb{N}}: \sum_{n} a_{n} e_{n} \text { converges in } X\right\} .
$$

Then $X_{0}$ is a subspace of $c_{0}$, non-closed in general. Define $T: X_{0} \rightarrow X$ by

$$
T\left(\left\{a_{n}\right\}\right)=\sum_{n=1}^{\infty} a_{n} e_{n} \quad \forall\left\{a_{n}\right\} \in X_{0}
$$

Then $T$ is linear and bijective. Furthermore, $T^{-1}(x)=\left\{f_{n}(x)\right\}_{n}$ for every $x \in X$. If $n \in \mathbb{N}$ and $x \in X$ we obtain

$$
\left|f_{n}(x)\right| \leq M\left\|f_{n}(x) e_{n}\right\|=M\left\|P_{n}(x)-P_{n-1}(x)\right\| \leq 2 K M\|x\|,
$$

where $\left\|e_{n}\right\| \geq 1 / M>0$ for all $n \in \mathbb{N}, K$ is the basic constant and $\left\{P_{n}\right\}$ is the sequence of the projections of the basis.

Thus, $T^{-1}$ is continuous. But $T\left(b_{n}\right)=e_{n}$ for all $n \in \mathbb{N}$, so $T$ is not continuous, that is, we can assume that for every $k \in \mathbb{N}$ there is $\left\{a_{n}^{k}\right\} \subset X_{0}$ such that $\lim _{k} a_{n}^{k}=0$ uniformly in $n \in \mathbb{N}$ and $\left\|\sum_{n=1}^{\infty} a_{n}^{k} e_{n}\right\| \geq 1$ for every $k \in \mathbb{N}$.

If we set $y_{p}=\sum_{n=1}^{\infty} a_{n}^{p} e_{n}$ for every $p \in \mathbb{N}$, we can apply Lemma 2 to obtain a basic block $\left\{v_{n}\right\}$ with $\inf _{n}\left\|v_{n}\right\|>0$ and $\lim _{k} \lambda_{k}=0$, where $v_{n}=\sum_{k=\sigma(n-1)+1}^{\sigma(n)} \lambda_{k} e_{k}$ for every $n \in \mathbb{N}$.

We put $Y=\overline{\operatorname{lin}}\left\{v_{n}: n \in \mathbb{N}\right\}$. Then $Y$ is not reflexive and $\lim _{n \rightarrow \infty}\left\|f_{n \mid Y}\right\|_{Y^{*}}$ $=0$, since $\lim _{k} \lambda_{k}=0$.

By Theorem 1, we conclude that $N_{X} \neq X$. 
Proof of Theorem A. The equivalence between (i) and (iii) is general and it is proved in Theorem 1 . The implications (i) $\Rightarrow$ (ii), (v) $\Rightarrow$ (iv) and (iv) $\Rightarrow$ (iii) are clear, and the implication $(\mathrm{i}) \Rightarrow(\mathrm{v})$ is Lemma 3 .

For (ii) $\Rightarrow$ (i), assume that $\left\{e_{n}\right\}$ is not supershrinking, so $N_{X} \neq X$. By Theorem 1 , there is a non-reflexive subspace $Y$ of $X$ such that $Y^{* *} \subset N_{X}$. Now, by Theorem 1.b.14 of [3], $Y^{* *}$ is not separable, since $X$ does not contain infinite-dimensional reflexive subspaces. Then $N_{X}$ is not separable.

Before proving Theorem $B$, we show what happens if one assumes the equality $N_{X}=X$ without $c_{0}$ copies.

Proposition 3. Let $X$ be a Banach space with a normalized basis $\left\{e_{n}\right\}$ without subspaces isomorphic to $c_{0}$ and assume that $N_{X}=X$. Then

(i) Every infinite-dimensional subspace of $X$ contains an infinite-dimensional reflexive subspace, that is, $X$ is somewhat reflexive.

(ii) Every subsequence of $\left\{e_{n}\right\}$ has a further subsequence whose closed linear span is a reflexive subspace.

Proof. (i) Let $Y$ be an infinite-dimensional subspace of $X$. Then by Proposition 1.a.11 of $[3]$, there is a basic sequence $\left\{y_{n}\right\}$ in $Y$ equivalent to a seminormalized basic block $v_{n}=\sum_{k=\sigma(n-1)+1}^{\sigma(n)} \lambda_{k} e_{k}$ for every $n \in \mathbb{N}$, with $\left\{\lambda_{k}\right\}$ bounded.

As $X$ does not contain $c_{0}$ copies, there is $\left\{t_{n}\right\} \subset c_{0}$ such that $\sum_{n} t_{n} v_{n}$ is not convergent.

Then there is $\varepsilon>0$ and natural numbers $p_{1}<q_{1}<\ldots<p_{n}<q_{n}<\ldots$ such that $\left\|\sum_{k=p_{n}}^{q_{n}} t_{n} v_{n}\right\| \geq \varepsilon$ for every $n \in \mathbb{N}$.

In this way, we obtain a basic block

$$
w_{n}=\sum_{k=\tau(n-1)+1}^{\tau(n)} \alpha_{k} e_{k}=\sum_{k=p_{n}}^{q_{n}} t_{k} v_{k} \quad \forall n \in \mathbb{N},
$$

with $\inf _{n}\left\|w_{n}\right\|>0$ and $\lim _{k} \alpha_{k}=0$.

Let $Z=\overline{\operatorname{lin}}\left\{w_{n}: n \in \mathbb{N}\right\}$. Then $\lim _{n \rightarrow \infty}\left\|f_{n \mid Z}\right\|_{Z^{*}}=0$, since $\lim _{k} \alpha_{k}=0$.

So, $Z$ is a reflexive subspace isomorphic to a subspace of $Y$, by Theorem 1 .

(ii) It is clear that it suffices to prove that $\left\{e_{n}\right\}$ has a subsequence whose closed linear span is a reflexive subspace.

For this, we apply the Elton Theorem to obtain a basic subsequence $\left\{e_{\sigma(n)}\right\}$ of $\left\{e_{n}\right\}$ such that

$$
\lim _{k}\left\|\sum_{i=1}^{k} a_{i} e_{\sigma(i)}\right\|=\infty \quad \forall\left\{a_{i}\right\} \notin c_{0} .
$$

We put $Y=\breve{l i n}\left\{e_{\sigma(n)}: n \in \mathbb{N}\right\}$. To see that $Y$ is reflexive it suffices to prove that $\left\{e_{\sigma(n)}\right\}$ is a boundedly complete basic sequence in $Y$. (Observe that the assumption $N_{X}=X$ implies that $\left\{e_{n}\right\}$ is shrinking.)
Let $\left\{\lambda_{n}\right\} \subset \mathbb{R}$ be such that $\sup _{n}\left\|\sum_{k=1}^{n} \lambda_{k} e_{\sigma(k)}\right\|<\infty$. Then $\left\{\lambda_{n}\right\} \in c_{0}$ and so $\sum_{n} \lambda_{n} e_{\sigma(n)}$ converges, that is, $Y$ is reflexive.

LEMMA 4. Let $X$ be a Banach space with a normalized basis $\left\{e_{n}\right\}$ and associated functionals $\left\{f_{n}\right\}$ such that $N_{X}=X$. If $v_{n}=\sum_{k=\sigma(n-1)+1}^{\sigma(n)} \lambda_{k} e_{k}$ is a basic block of $\left\{e_{n}\right\}$ with $\left\{\lambda_{n}\right\}$ bounded and $Y=\varlimsup \operatorname{lin}\left\{v_{n}: n \in \mathbb{N}\right\}$, then $N_{Y}=Y$

Proof. Let $\left\{g_{n}\right\}$ be the sequence of associated functionals of $\left\{v_{n}\right\}$. Then $f_{k}=\lambda_{k} g_{n}$ whenever $\sigma(n-1)+1 \leq k \leq \sigma(n)$.

If $y^{* *} \in Y^{* *}$ with $\lim _{n} y^{* *}\left(g_{n}\right)=0$ then $\lim _{n} y^{* *}\left(f_{n}\right)=0$, since $\left\{\lambda_{n}\right\}$ is bounded. So, $y^{* *} \in N_{X}=X$ and $N_{Y}=Y$.

Proof of Theorem $B$. Let $Z$ be a non-reflexive subspace of $X$. Then, by Proposition 1.a.11 of [3], it is possible to find a basic sequence $\left\{z_{n}\right\}$ in $Z$ which is weakly Cauchy, not weakly convergent, and whose difference sequence $\left\{z_{n+1}-z_{n}\right\}$ is equivalent to a seminormalized block basis of $\left\{e_{n}\right\}$. The subspace generated by the sequence $\left\{z_{n+1}-z_{n}\right\}$ is non-reflexive and satisfies the same hypotheses as $X$, by Lemma 4 , so it is sufficient to see that $X$ contains an order-one quasireflexive subspace if $X$ is not reflexive.

So, assume that $X$ is not reflexive and let $x_{0}^{* *} \in X^{* *} \backslash X$. Now, the sequence $\left\{\sum_{k=1}^{n} x_{0}^{* *}\left(f_{k}\right) e_{k}\right\}_{n}$ is weakly Cauchy and not weakly convergent. By the $c_{0}$-Rosenthal Theorem we find $\sigma: \mathbb{N} \rightarrow \mathbb{N}$ increasing such that the sequence

$$
y_{n}=\sum_{k=1}^{\sigma(n)} x_{0}^{* *}\left(f_{k}\right) e_{k}
$$

is basic and strongly summing. We put $Y=\varlimsup i n\left\{y_{n}: n \in \mathbb{N}\right\}$. We can suppose that $x_{0}^{* *}\left(f_{\sigma(n)}\right) \neq 0$ for all $n \in \mathbb{N}$.

Let $\left\{g_{n}\right\}$ be the sequence of associated functionals of $\left\{y_{n}\right\}$. Then

$$
g_{n}=\frac{f_{\sigma(n) \mid Y}}{x_{0}^{* *}\left(f_{\sigma(n)}\right)}-\frac{f_{\sigma(n+1) \mid Y}}{x_{0}^{* *}\left(f_{\sigma(n+1)}\right)} \quad \forall n \in \mathbb{N} .
$$

If $y^{* *} \in Y^{* *}$ then $\sup _{n}\left\|\sum_{k=1}^{n} y^{* *}\left(g_{k}\right) y_{k}\right\|<\infty$. So, the series $\sum_{n} y^{* *}\left(g_{n}\right)$ converges. Therefore, the following limit exists, for every $y^{* *} \in Y^{* *}$ :

$$
\lim _{n \rightarrow \infty} \frac{y^{* *}\left(f_{\sigma(n)}\right)}{x_{0}^{* *}\left(f_{\sigma(n)}\right)}
$$

We put $v_{n}=\sum_{k=\sigma(n-1)+1}^{\sigma(n)} x_{0}^{* *}\left(f_{k}\right) e_{k}$ and $h_{n}=f_{\sigma(n)} / x_{0}^{* *}\left(f_{\sigma(n)}\right)$ for every $n \in \mathbb{N}$. Hence $\left\{v_{n}\right\}$ is a shrinking basis of $Y$ with associated functionals $\left\{h_{n}\right\}$. 
Finally, we define $\phi: Y^{* *} \rightarrow \mathbb{R}$ by $\phi\left(y^{* *}\right)=\lim _{n} y^{* *}\left(h_{n}\right)$. By (3), we have $\phi \in Y^{* * *}$. Now, by applying Lemma 4 ,

$$
Y^{* *}=\operatorname{Ker} \phi \oplus\left\langle x_{0}^{* *}\right\rangle=N_{Y} \oplus\left\langle x_{0}^{* *}\right\rangle=Y \oplus\left\langle x_{0}^{* *}\right\rangle .
$$

Thus $Y$ is an order-one quasireflexive subspace.

REMARK. With an analogous proof, the conclusion of Theorem $B$ is also true if we suppose that $\operatorname{dim}\left(N_{X} / X\right)<\infty$ instead of $N_{X}=X$.

Acknowledgements. This work was done while the author visited University Paris VI for a research stay. I want to thank Professor G. Godefroy for his invitation and his kindness during this stay. Also, I wish to thank Professor E. Odell for his indications concerning Proposition 3(ii). Finally, I want to thank the referee for his addition to the proof of Theorem B.

\section{References}

1] J. Diestel, Sequences and Series in Banach Spaces, Springer, 1984

2] Ghoussoub and B. Maurey, $G_{\delta}$-embeddings in Hilbert space, J. Funct. Anal. 61 (1985), $72-97$.

[3] J. Lindenstrauss and L. Tzafriri, Classical Banach Spaces I, Ergeb. Math. Grenzgeb. 92, Springer, 1977.

[4] G. López and J. F. Mena, RNP and KMP are equivalent for some Banach spaces with shrinking basis, Studia Math. 118 (1996), 11-17.

5] H. Rosenthal, A subsequence principle characterizing Banach spaces containing $c_{0}$ Bull. Amer. Math. Soc. 30 (1994), 227-233.

[6] -, Boundedly complete weak-Cauchy sequences in Banach spaces, preprint.

Departamento de Análisis Matemático

Facultad de Ciencias

Universidad de Granada

18071 Granada, Spain

E-mail: glopezp@goliat.ugr.es

\section{A spectral theory for locally compact abelian groups of automorphisms of commutative Banach algebras}

by

SEN-ZHONG HUANG (Jena and Rostock)

Abstract. Let $\mathcal{A}$ be a commutative Banach algebra with Gelfand space $\Delta(\mathcal{A})$. Denote by $\operatorname{Aut}(\mathcal{A})$ the group of all continuous automorphisms of $\mathcal{A}$. Consider a $\sigma(\mathcal{A}, \Delta(\mathcal{A})$ )continuous group representation $\alpha: G \rightarrow \operatorname{Aut}(\mathcal{A})$ of a locally compact abelian group $G$ by automorphisms of $\mathcal{A}$. For each $a \in \mathcal{A}$ and $\varphi \in \Delta(\mathcal{A})$, the function $\varphi_{a}(t):=\varphi\left(\alpha_{t} a\right)$ $(t \in G)$ is in the space $C(G)$ of all continuous and bounded functions on $G$. The weak-star spectrum $\sigma_{w^{*}}\left(\varphi_{a}\right)$ is defined as a closed subset of the dual group $\widehat{G}$ of $G$. For $\varphi \in \Delta(\mathcal{A})$ we define $\Lambda_{\varphi}^{\alpha}$ to be the union of all sets $\sigma_{w^{*}}\left(\varphi_{a}\right)$ where $a \in \mathcal{A}_{1}$ and $\Lambda_{\alpha}$ to be the closure of the union of all sets $\Lambda_{\varphi}^{\alpha}$ where $\varphi \in \Delta(\mathcal{A})$, and call $\Lambda_{\alpha}$ the unitary spectrum of $\alpha$.

Starting by showing that the closure of $\Lambda_{\varphi}^{\alpha}$ (for fixed $\varphi \in \Delta(\mathcal{A})$ ) is a subsemigroup of $\widehat{G}$ we characterize the structure properties of the group representation $\alpha$ such as norm continuity, growth and existence of non-trivial invariant subspaces through its unitary spectrum $\Lambda_{\alpha}$.

For an automorphism $T$ of a semisimple commutative Banach algebra $\mathcal{A}$ we consider the group representation $\mathbf{T}: \mathbb{Z} \rightarrow \operatorname{Aut}(\mathcal{A})$ defined by $\mathbf{T}_{n}:=T^{n}$ for all $n \in \mathbb{Z}$. It is shown that $\Lambda_{\mathfrak{T}}=\sigma(T) \cap \mathbb{T}$, where $\sigma(T)$ is the spectrum of $T$ and $\mathbb{T}$ is the unit circle. From this fact we give an easy proof of the Kamowitz-Scheinberg theorem which asserts that the spectrum $\sigma(T)$ either contains $\mathbb{T}$ or is a finite union of finite subgroups of $\mathbb{T}$.

Introduction. Let $\mathcal{A}$ be a commutative Banach algebra with Gelfand space $\Delta(\mathcal{A})$ (i.e., the space of regular maximal ideals of $\mathcal{A}$ ). Denote by Aut $(\mathcal{A})$ the group of all continuous automorphisms of $\mathcal{A}$. For an automorphism $T$ on $\mathcal{A}$ we consider the group representation $\mathbf{T}: \mathbb{Z} \rightarrow \operatorname{Aut}(\mathcal{A})$ given by $\mathbf{T}_{n}:=T^{n}$ for all $n \in \mathbb{Z}$. For each $a \in \mathcal{A}$ and $\varphi \in \Delta(\mathcal{A})$ the function $\varphi_{a}(n):=\varphi\left(T^{n} a\right)(n \in \mathbb{Z})$ belongs to the space $C(\mathbb{Z})$ of all continuous and bounded functions on the group $\mathbb{Z}$. The weak-star spectrum $\sigma_{w^{*}}\left(\varphi_{a}\right)$ of $\varphi_{a}$, as a closed subset of the unit circle $\mathbb{T}$, is defined in the classical way (see [14] or [20]). Note that $\mathbb{T}$ is the dual group of $\mathbb{Z}$.

1991 Mathematics Subject Classification: Primary 46J05, 47A10; Secondary 43A22, 47 A11.

Key words and phrases: automorphism, group representation, spectral analysis. 